\title{
Résultats d'essais sur le canal de la chute de Palaminy (Haute-Garonne)
}

\section{Results of recent tests on the Palaminy canal (Haute-Garonne Department)}

\author{
PAR L. CASTEX, \\ DIRECTEUR TECHNIQUE AUX LABORATOIRES D'HYDRAULIQUE \\ DE L'INSTITUT DE MÉCANIQUe DES FLUIDES
}

R. MATHALY,

INGÉNIEUR AU SERVICE ÉTUDES

DE LA RÉGION D'ÉQUIPEMENT HYDRAULIQUE GARONNE ÉLECTRICITÉ DE FRANCE

ET
C. THIRRIOT,

MAÎTRE DE CONFÉRENCES A LA Faculte DES SCIENCES DE TOUlouse

\begin{abstract}
Essais systématiques effectués, tant en régime permanent qu'en régime transitoire, conjointement entre les Services Techniques de la Région d'Equipement Hydraulique Garonne d'Electricité de France et le Laboratoire d'Hydraulique de l'Ecole Nationale Supérieure d'Electrotechnique et d'Hydraulique de Toulouse.

Procédés spéciaux conduisant à une excellente précision tant pour la détermination des coefficients de perte de charge que pour l'enregistrement des fronts d'intumescence.

En ce qui concerne le premier point, les essais effectués d̀ plusieurs mois d'intervalle ont mis en évidence un «vieillissement» important du canal inexplicable par l'aspect extérieur des parois.

Méthodes opératoires adoptées, résultats obtenus et comparaison de ces derniers avec les prévisions.
\end{abstract}

\begin{abstract}
Systematic tests at both steady and transient flow conditions have been jointly carried out by the technical department of the "Region d'Equipement Hydraulique Garonne" of "Electricite de France" and the hydraulics laboratory of the "Ecole Nationale Superieure d'Electrotechnique, d'Electronique et d'Hydraulique" of Toulouse.

Special methods have enabled a very high degree of accuracy to be achieved in determining the head loss coefficients and recording wave fronts. In the former connection, tests carried out at several months interval showed that the canal had "aged" considerably, though the external appearance of its walls gave no indication as to why this should have happened. The operating methods used and results obtained are described, and the latter are compared with the predicted data.
\end{abstract}

\section{I. - INTRODUCTION}

Depuis de nombreuses années, les Ingénieurs chargés de l'étude d'un ouvrage à surface libre se posent la question du choix de la formule à adopter pour le calcul de la perte de charge ainsi que du coefficient numérique intervenant dans ces formules.
Dans la littérature technique, on ne trouve guère de renseignements basés sur des mesures précises effectuées sur des ouvrages industriels, contrairement à ce qui existe pour les ouvrages en charge, pour lesquels de nombreux comptes rendus sont donnés. 
Nous pensons qu'il faut voir, dans cet état de fait, les difficultés réelles que l'on éprouve à effectuer des mesures ayant un certain caractère de précision sur des ouvrages de grande dimension, pour lesquels il est assez difficile d'obtenir une stabilité de fonctionnement de durée suffisante : permanence du débit, constance du tirant d'eau à l'origine de l'ouvrage. C'est la raison pour laquelle nous avons pensé qu'il serait intéressant de relater les essais effectués sur le canal de la chute de Palaminy. Ces essais ont en effet été réalisés dans des conditions exceptionnelles en raison même du but recherché qui était la comparaison de la perte de charge entre deux troncons du canal : l'un revêtu en béton bitumineux (1), l'autre revêtu en béton par des procédés spéciaux «vacuum concrete $\gg$.

\section{II. - RAPPEL DES CARACTERISTIQUES ESSENTIELLES DU CANAL D'AMENÉE}

- longueur : $5725 \mathrm{~m}$;

- section trapézoïdale avec largeur radier : $5,50 \mathrm{~m}$ et fruit de bajoyers $3 / 2$;

- pente du radier : $0,15 \%$;

- cote du radier à l'origine du canal au PK 165 : $252,65 \mathrm{~m}$ NGF.

Le canal d'amenće est entièrement revètu en béton, à l'exclusion d'un troncon de $400 \mathrm{~m}$ de longueur sur lequel le revêtement est en béton bitumineux. Ce troncon se situe entre les PK 517 et $917 \mathrm{~m}$.

L'épaisseur du revêtement béton bitumineux est de $0,09 \mathrm{~m}$. Il en résulte un léger décrochement au passage du troncon normal au troncon bitumineux, ainsi qu'un léger accroissement de section de ce dernier pour un même tirant d'eau. Les résultats tiennent compte de cette différence. Par contre, le radier est continu et ne présente aucun décrochement.

\section{III. - DISPOSITIF EXPÉRIMENTAL}

Pour un fonctionnement du canal en régime permanent établi, le coefficient de perte de charge se déduit de la connaissance :

- des caractéristiques géométriques du canal;

- du débit;

- du profil en long de la ligne d'eau dans chacun des tronçons soumis aux essais.

\section{Mesure du débit de régime permanent.}

Au cours des essais de perte de charge, la mesure du débit dans le canal a pu être réalisée dans des circonstances tout à fait exceptionnelles, en ce qui concerne la précision.

En effet, ces essais ont été effectués immédiatement à la suite des essais de rendement des groupes.

Au cours de ces derniers, les débits ont élé mesurés sur le groupe $\mathrm{n}^{\circ} 2$, au moyen d'une batterie de moulinets disposés sur un croisillon dans la conduite forcée. Ces mesures de débit

(1) Référence: article de M. Vatlée dans la rẹvue Construction de juin 1960 . ont permis d'effectuer l'étalonnage du dispositif de mesure «Winter Kennedy», aménagé sur la bâche spirale de la turbine. Ce dispositif comprend quatre couples de prises de pression ayant đonné lieu chacun à un étalonnage.

\section{A - DÉbit ABSORBÉ PAR LE GROUPE 2 :}

Pour les essais de perte de charge, le débit absorbé par le groupe 2 a été déterminé au moyen du dispositif «Winter Kennedy»; simultanément, à titre de recoupement, on a mesuré la puissance aux bornes du groupe ainsi que la hauteur de chute nette. Connaissant le rendement global du groupe, une deuxième valeur du débit a pu être déterminée à partir de ces éléments.

\section{B - Débit abSORBÉ PAR LE GROUPE 1:}

Lors des essais de réception des turbines, seul le rendement du groupe 2 a été mesuré.

Nous avons admis que, dans les mêmes conditions de fonctionnement, le rendement du groupe 1 est identique à celui du groupe 2 , et nous avons déduit de cette hypothèse, au cours d'essais spéciaux, les valeurs des coefficients 
$\mathrm{K}_{1}, \mathrm{~K}_{2}, \mathrm{~K}_{3}$, et $\mathrm{K}_{4}$ correspondant à chacun des couples «Winter Kennedy» installés sur le groupe 1 en supposant que la relation liant le débit $Q$ à la différence de pression $\Delta h$ relative à cette courbe est de la forme :

$$
\Delta h=\mathrm{K}_{n} \mathrm{Q}^{2}
$$

Partant de ces valeurs ainsi déterminées, nous avons repris entièrement un essai de rendement pour le groupe 1 , qui a conduit à une enveloppe des rendements maximaux ne présentant pas d'écart supérieur à $0,5 \%$ par rapport à la courbe enveloppe des maximums des rendements relevés sur le groupe 2.

A titre de recoupement, au cours des essais de perte de charge, il a également été relevé Ies valeurs de la puissance aux bornes et de la chute nette.

\section{C - Remarque concernant laA prÉcision des MESURES DE DÉBIT.}

Etant domné la présence d'une conduite forcée alimentant les groupes, la précision de la mesure du débit ayant permis l'étalonnage du dispositif «Winter Kennedy» du groupe 2 est excellente. Il ressort d'un calcul d'erreur, dont nous ne donnerons pas le détail, que l'erreur maximale commise sur la détermination du débit au moyen des moulinets est de l'ordre de $0,3 \%$.

L'erreur commise sur le débit mesuré à chacun des couples W.K. ne dépasse pas $1 \%$, de telle sorte que la moyenne des mesures sur les quatre couples correspond à une précision de $0,5 \%$, ce qui conduit à une erreur sur la con- naissance de débit absorbé par le groupe 2 ne dépassant pas $0,8 \%$.

L'erreur commise sur la mesure du débit du groupe 1 est analogue à l'erreur commise sur la mesure du débit du groupe 2, sauf à y adjoindre l'imprécision due à l'hypothèse de base concernant l'identité du rendement des deux groupes. Les essais de rendement nous ont montré que nous pouvions évaluer à $0,5 \%$ l'écart entre les groupes. D'où il ressort que l'erreur relative sur le débit du groupe 1 est égale à $0,8 \%$ plus 0,5 , soit $1,3 \%$. En définitive, le débit total est connu avec une précision de :

$$
\frac{0,8}{2}+\frac{1,3}{2} \# 1 \%
$$

\section{Détermination du profil en long de la ligne d'eau dans chacun des tronçons soumis aux essais.}

Il s'agit de déterminer, entre les PK 417 et 1417 , les cotes du plan d'eau moyen dans diffèrentes sections du canal au cours d'un fonctionnement en régime permanent.

Après une étude approfondie du problème avant la construction du canal, nous avons proposé la mise en place de dispositifs spéciaux de mesure du tirant d'eau, en vue d'obtenir la précision requise pour que de tels essais aient une signification. La mise en place de ces dispositifs a été faite durant les travaux. Nous avons choisi quatorze sections de mesures, dont sept dans le tronçon bitumineux correspondant à une section

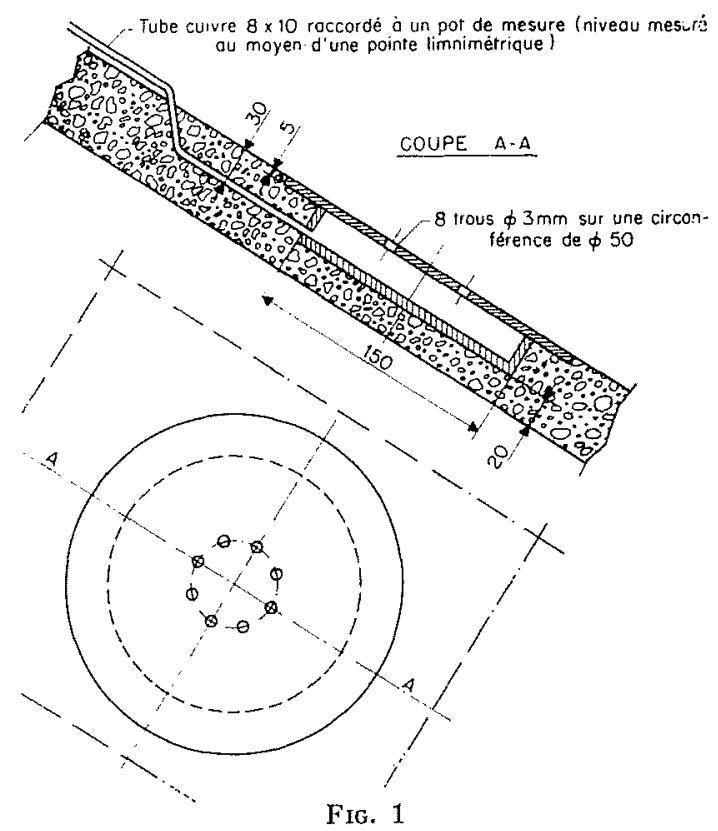

Détail d'une prise de pression arasée au bajoyer à $1 \mathrm{~m}$ aundessus du radier. 


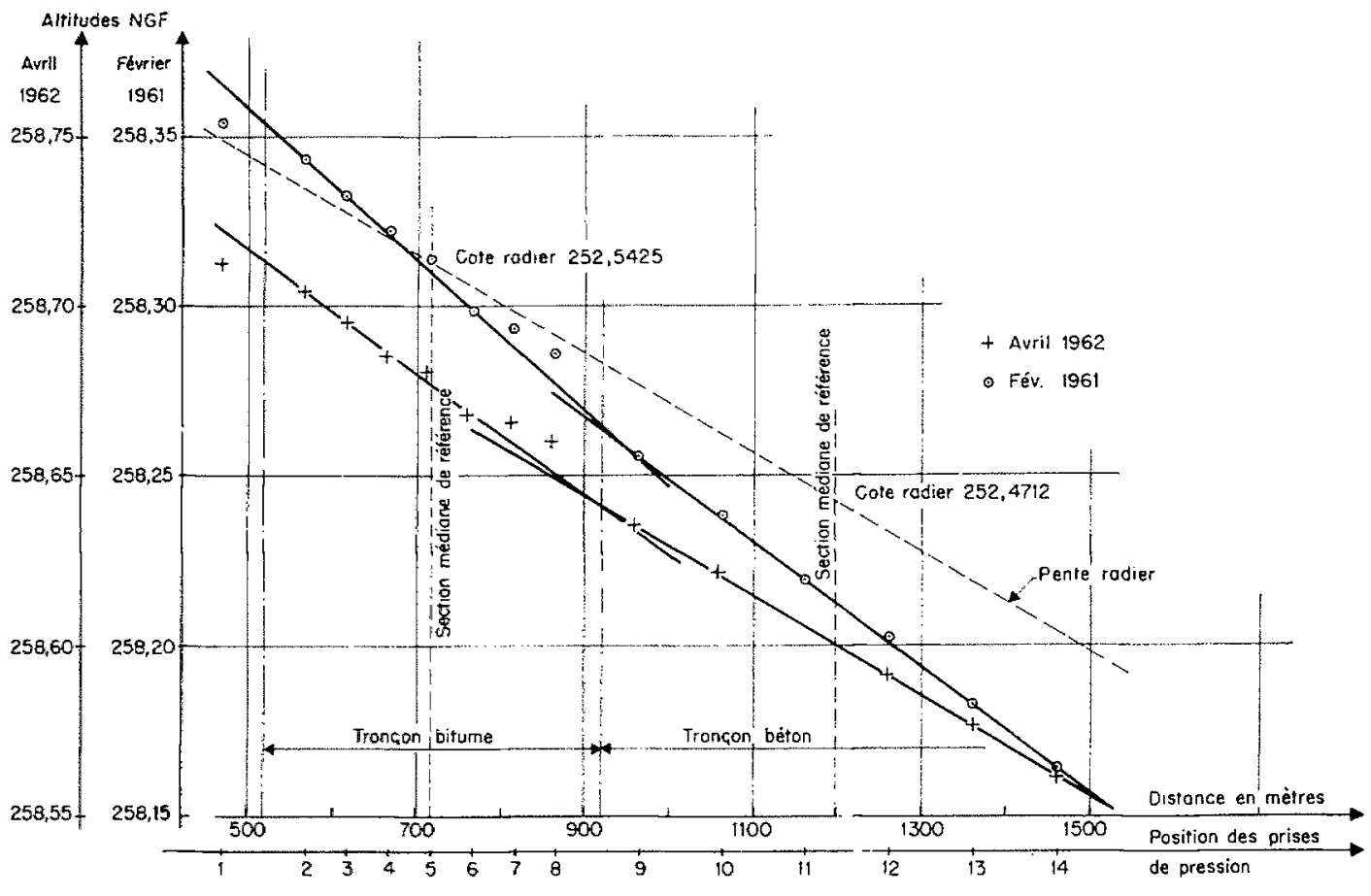

FIG. 2

Exemples de relevés de la ligne d'eau. Mesures $n^{0} 6$.

tous les $50 \mathrm{~m}$, et sept dans le tronçon béton correspondant à une section tous les $100 \mathrm{~m}$. L'emplacement de ces sections de mesure est précisé sur la figure 2.

Chacune de ces sections est équipée de deux prises de pression constituées par une boîte en bronze scellée dans les bajoyers du canal. Ces prises sont reliées à des pots de mesures par l'intermédiaire de tubes en polyvinyle. Des pointes limnimétriques permettent la mesure des niveaux dans les pots de mesure. La figure 1 précise les dispositions adoptées.

Un nivellement exécuté dans les meilleures conditions à l'aide d'un niveau Wild N3 et d'une mire en métal invar, a permis de relier le zéro des limnimètres à pointe au NGF et de déduire ainsi, de la mesure dans les pots, l'altitude de la ligne d'eau en chaque point de mesure.

\section{IV. - REALISATION DES ESSAIS}

\section{Réglage préalable.}

La durée de chaque essai a été de 6 à 7 h environ, au cours de laquelle on s'est efforcé de maintenir le régime permanent aussi bien que possible. Le régime permanent dépend :

- de la constance du débit absorbé par les groupes;

- de la constance du niveau dans la retenue du barrage.

Afin d'obtenir la meilleure précision possible, les essais ont été réalisés au voisinage de la pleine ouverture des deux groupes correspon- dant au débit maximal absorbé. Le régulateur des turbines n'a pas eu à intervenir et, au cours de chaque essai, l'ouverture des groupes et la puissance aux bornes est demeurée rigoureusement constante.

Le réglage du niveau dans la retenue a été réalisé par manœuvre $\dot{a}$ main d'un clapet du barrage.

Nous avons utilisé, pour la détection du niveau amont, le dispositif Saint-Chamond (puits et flotteur) du transmetteur de niveau à l'usine. Un repère fixe devant la poulie du transmetteur nous permettait de déceler la moindre variation, 
corrigée aussitôt par des manœuvres du clapet fréquentes et de petite amplitude.

Le niveau a été ainsi maintenu constant à 1 ou $2 \mathrm{~mm}$ près, pendant la durée de chaque essai.

\section{Exécution des relevés.}

Pour chaque essai, les relevés ont été exécutés après deux ou trois heures de stabilisation du régime.

a) A l'usine, un opérateur notait pour chaque groupe : la puissance aux bornes, l'ouverture du distributeur, l'ouverture de la roue, la pression différentielle correspondant à chaque couple du dispositif Winter Kennedy, la cote amont, la cote du niveau aval.

b) Au barrage, l'opérateur chargé du réglage de la retenue notait la cote relative du plan d'eau, en fonction du temps, à raison d'une lecture toutes les deux minutes environ.

c) Le long du canal, quatre opérateurs notaient, en fonction du temps, les lectures aux différents limnimètres : six à huit lectures ont été faites sur chaque limnimètre en prenant soin que chacun d'eux ait été relevé au moins une fois par chaque opérateur.

Pour chaque essai, les relevés ont été poursuivis pendant deux heures au minimum.

\section{V. - RESULTATS}

\section{Résultats bruts.}

Première série d'essais (janvier 1961):

Sept essais ont été effectués pour des débits sensiblement identiques, mais pour des niveaux au barrage variant entre le niveau de retenue normal $259,40 \mathrm{~m}$ et le niveau minimal autorisé $258,60 \mathrm{~m}$.

Pour chaque limnimètre, il a été tracé la courbe du niveau en fonction du temps. Toutes ces courbes ont des allures semblables et présentent un maximum et un minimum. Nous avons adopté comme niveau, pour le tracé de la ligne d'eau, celui correspondant au maximum ou au minimum, suivant le cas, se produisant au même instant. On est assuré, dans ces conditions, de tenir compte du point pour lequel le régime est le plus stable.

La. figure 2 donne un exemple de tracé de la ligne d'eau (mesure $n^{\circ} 6$ ). Le tableau I résume les résultats obtenus.

\section{Deuxième série d'essais (avril 1962):}

Neuf essais ont été effectués. Les deux premiers ont été éliminés d'office en raison du vent qui régnait lors des mesures et qui n'a pas permis, par la suite, un tracé correct des lignes d'eau dans le canal.

Nous avons donc retenu les mesures $n^{\text {os }} 3 \dot{a} 9$ effectuées pour des débits compris entre 123 et

\section{Tableat I}

Première série d'essais (janvier 1961)

\begin{tabular}{|c|c|c|c|c|c|c|c|c|c|}
\hline \multicolumn{2}{|l|}{ NUMÉro de L'ESSAI } & 1 & 2 & $y$ & 4 & 5 & 6 & 7 & \multirow{2}{*}{$\begin{array}{l}\text { VALEURS } \\
\text { MOYENNES }\end{array}$} \\
\hline Débit canal & $\mathrm{m}^{3} / \mathrm{s}$ & 141,7 & 165 & 166,8 & 166,6 & 166,1 & 165,2 & 162,8 & \\
\hline \multirow{2}{*}{ Coefficient de Strickler } & Béton & 73,5 & 67,87 & 68,44 & 68,99 & 68,8 & 71,68 & 68,69 & 69,6 \\
\hline & Bitume & 63,37 & 63,47 & 65,29 & 64,36 & 63,73 & 64,07 & 63,32 & 63,9 \\
\hline \multirow{2}{*}{ Coefficient de Chézy. } & Béton & 90,6 & 83,5 & 84,2 & 84,0 & 84,0 & 86,5 & 82,7 & 84,7 \\
\hline & Bitune & 77,9 & 78,0 & 80,4 & 77,4 & 76,5 & 77,2 & 76,2 & 77,2 \\
\hline \multicolumn{2}{|c|}{ Coefficient de pondération...... } & 1 & 1 & 1 & 3 & 3 & 3 & 3 & \\
\hline
\end{tabular}


Tableau II

Deuxième série d'essais (avril 1962)

\begin{tabular}{|c|c|c|c|c|c|c|c|c|c|}
\hline \multicolumn{2}{|l|}{ Numéro de L'Essai } & \multirow{2}{*}{$\begin{array}{c}3 \\
151,55\end{array}$} & \multirow{2}{*}{$\begin{array}{c}4 \\
159,93\end{array}$} & \multirow{2}{*}{$\begin{array}{c}5 \\
163,91\end{array}$} & \multirow{2}{*}{$\begin{array}{c}6 \\
153,78\end{array}$} & \multirow{2}{*}{$\begin{array}{c}7 \\
142,92\end{array}$} & \multirow{2}{*}{$\begin{array}{c}8 \\
123,92\end{array}$} & \multirow{2}{*}{$\begin{array}{c}9 \\
145,77\end{array}$} & \multirow{2}{*}{$\begin{array}{l}\text { VALEURS } \\
\text { MOXENNES }\end{array}$} \\
\hline Débit canal & $\mathrm{m}^{3} / \mathrm{s}$ & & & & & & & & \\
\hline \multirow{2}{*}{ Coefficient de Strickler } & Béton & 62,01 & 62,89 & 66,31 & 63,88 & 62,14 & 63,77 & 62,63 & 63,4 \\
\hline & Bitume & $56,0 \mathrm{~S}$ & 56,90 & 57,71 & 56.51 & 58,90 & 55,05 & 58,20 & 57,1 \\
\hline \multirow{2}{*}{ Coefficient de Chézy. } & Béton & 75,94 & $75, \overline{55}$ & 80,03 & 77,82 & 76,43 & 78,28 & 77,05 & 77,3 \\
\hline & Bitume & 68,74 & 68,53 & 69,79 & 68,90 & 72,48 & 67,58 & 71,63 & 69,4 \\
\hline \multicolumn{2}{|c|}{ Coefficient de pondération...... } & 2 & 2 & 2 & 4 & 2 & 1 & 2 & \\
\hline
\end{tabular}

$164 \mathrm{~m}^{3} / \mathrm{s}$ et pour des niveaux, an barrage, variant de la cote maximale 259,39 à la cote minimale possible $258,62 \mathrm{~m}$.

Le tableau II résume les résultats obtenus.

Nous jugeons intéressant de donner un deuxième exemple (fig. 2) de tracé de la ligne d'eau correspondant à l'essai $n^{\circ} 6$, pour montrer que la dispersion des points expérimentaux, par rapport à la courbe moyenne, est faible et que les mêmes anomalies se retrouvent à un an d'intervalle sur les mêmes prises de pression. Il est donc possible que les prises correspondantes ou bien soient influencees par le changement de section dù à la discontinuité du revêtement du canal, ou bien soient mal disposées sur la paroi du canal.

\section{Calcul des coefficients de pertes de charge.}

Les mesures effectuées donnent pour chaque essai, le débit et la ligne d'eau correspondante en régime permanent.

La portion du canal intéressée étant relativement courte, la courbe de remous peut être considérée comme rectiligne le long des deux tronçons de mesure.

La variation du tirant d'eau $h$ est donnée par l'expression classique de l'écoulement varié :

$$
\frac{d h}{d x}=\frac{\mathrm{I}-\mathrm{J}}{1-\left(v^{2} / c^{2}\right)}
$$

dans laquelle :

$J$ est la pente de la ligne de charge;

$v$ la vitesse moyenne des molécules de fluides dans le profil considéré; c la célérité des ondes superficielles.

Posons :

$$
\eta=\frac{v^{2}}{c^{2}}
$$

Par ailleurs :

$$
\frac{d h}{d x}=\mathrm{I}-i
$$

$i$ étant la perte superficielle longitudinale.

$$
\mathrm{J}=(1-\eta) i+\eta \mathbf{I}
$$

Si le régime d'écoulement est uniforme, c'est-à-dire $i=I$, on retrouve l'expression simplifiée :

$$
\mathrm{J}=i
$$

L'écart d'avec cette valeur dépend du nombre de Froude $v / c$. Plus l'écoulement sera proche du régime critique et plus l'écart sera important.

A partir des valeurs expérimentales, le coefficient $K$ est donné par la formule de Strickler.

$$
\mathrm{K}==\frac{\mathrm{V}}{\mathrm{R}^{2 / 3} \mathrm{~J}^{1 / 2}}
$$

Le coefficient de Chézy aurait pour valeur

$$
\mathrm{C}=\mathrm{KR}^{\mathrm{1} / 6}
$$

Les tableaux I et II présentent le résultat des calculs.

Les valeurs moyennes obtenues à partir des résultats des essais effectués sont indiqués dans le tableau III : 


\section{TABLEAU III}

\begin{tabular}{|c|c|c|c|c|c|c|}
\hline \multirow[b]{3}{*}{ Coefficient de Strickler. . . . K } & \multicolumn{3}{|c|}{ TRONĢON BÉTON } & \multicolumn{3}{|c|}{ 'TRONGON BITUMF } \\
\hline & 1961 & 1962 & Ecart $\%$ & 1961 & 1962 & Ecart \% \\
\hline & 69,6 & 63,4 & $-8,9$ & 63,9 & 57,1 & $-10,6$ \\
\hline Coefficient de Chézy. ...... C & 84,7 & 77,3 & $-8,7$ & 77,2 & 69,1 & $-10,1$ \\
\hline
\end{tabular}

\section{VI. - REMARQUE CONCERNANT LA PRÉCISION DES MESURES}

Deux procédés sont possibles pour évaluer l'erreur commise sur le calcul du coefficient de Strickler :

- une méthode que l'on peut qualifier de statique, et qui consiste en l'étude de la répartition des valeurs obtenues pour $K$. Elle conduit à une erreur de $1,4 \%$ sur la valeur moyenne de $K$, à laquelle on doit ajouter la valeur de l'erreur systématique sur le débit, soit $0,5 \%$.

- une méthode plus physique, fondée sur l'évaluation directe de l'erreur relative maximale compte tenu des erreurs systématiques et accidentelles commises sur la détermination des différentes grandeurs intervenant dans la définition de K. La conjonction des différentes causes d'erreur conduit à un écart relatif de $3,85 \%$ sur une mesure. Mais le paramètre de précision de l'ensemble de $n$ mesures est multiplié par $\sqrt{n}$. Ainsi, l'erreur sur la moyenne est de l'ordre de $1,45 \%$.

En conclusion, l'erreur sur $K$ est inférieure à $2 \%$.

\section{VII. - CRITIQUE DES RESULTATS}

Le but des essais était essentiellement de comparer les coefficients de rugosité d'un tronçon revêtu en béton par le procédé «vacuum concrete ».

Les résultats montrent que le coefficient $K$ de Strickler est supérieur dans ce dernier tronçon, l'écart relatif étant d'environ $10 \%$ pour les deux séries d'essais.

Cet écart est largement significatif, car il demeurerait toujours important même si les imprécisions de-mesure intervenaient avec des signes opposés sur chaque tronçon. L'examen de la figure 2 montre nettement le changement de pente de la ligne piézométrique au droit de la discontinuité du revêtement.

Par ailleurs, le coefficient $K$ relatif au revêtement béton est nettement supérieur à la valeur habituellement admise dans la première série d'essais, ce qui s'explique par le procédé de construction utilisé pour le revêtement, d'une part du fait du réglage soigné des terrassements et, d'autre part, du procédé de mise en place du béton, procédé «vacuum concrete».

La deuxième série d'essais, intervenue quinze mois plus tard, a montré une évolution importante des coefficients de rugosité, matérialisée par une augmentation des pertes de charge de l'ordre de $20 \%$.

$\mathrm{Ce}$ «vieillissement» rapide nous a incité à examiner la rugosité absolue d'après les formules modernes de pertes de charge suivantes:

- formule de Colebrook :

$$
\frac{1}{\sqrt{\lambda}}=-2 \log \left(\frac{\varepsilon}{3,7 \mathrm{D}}+\frac{2,51}{\alpha \sqrt{\lambda}}\right)
$$

en admettant que cette formule, établie pour les conduites à section circulaire, est encore valable dans le cas de trapézoïdaux. 
- formule de Powell :

$$
\mathrm{C}=-23,3 \log \left(\frac{1,8 \mathrm{C}}{a}+\frac{\varepsilon}{\mathrm{R}}\right)
$$

-.- formule simplifiée :

$$
K=\frac{26}{\varepsilon^{1 / 6}}
$$

formules dans lesquelles :

$\varepsilon$ est la rugosité absolue des parois;

$\lambda$ coefficient universel des pertes de charge, tel que : $\lambda=\left(8 g / \mathrm{C}^{2}\right)$;

$C$ coefficient de Chézy;

$\mathrm{K}$ coefficient de Strickler;

$\mathrm{R}$ rayon hydraulique;

$\mathrm{D}=4 \mathrm{R}$ diamètre équivalent;

$a$ nombre de Reynolds.

Le tableau IV résume les résultats obtenus par l'application de ces formules.

Les valeurs de $\varepsilon$ trouvées semblent plausibles, mais sont nettement différentes selon la formule utilisée.

Ceci montre la difficulté que l'on rencontre pour l'application de ces formules, faisant intervenir un paramètre $\varepsilon$ que notts ne savons ni définir, ni mesurer avec la précision désirable.

Nous avons cependant envisagé différentes hypothèses pour expliquer ce «vieillissement \$ des parois.

L'observation visuelle des parois du canal ne permet de déceler aucun changement de l'état de surface. On peut toutefois émettre l'hypothèse de la présence de fines algues, accrochées à la paroi, qui vibreraient dans l'écoulement et qui, dès l'instant où le canal est mis à sec pour une visite, sèchent et demeurent pratiquement invisibles.

Par contre, un examen minutieux de l'état des joints dans le tronçon de béton fait apparaître une déformation des joints que l'on peut schématiser sur la figure 3 .

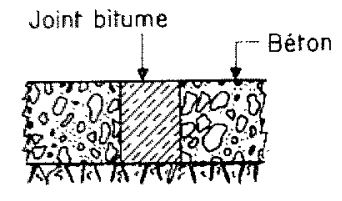

Etat neut

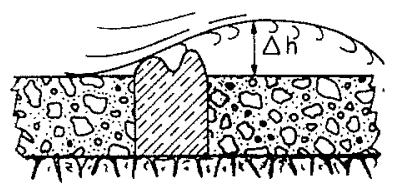

Etat actuel
Fig. 3

Un calcul fondé sur la base d'une perte à la Borda conduit à une majoration de perte de charge de $2 \%$ pour $\Delta h=1 \mathrm{~cm}$ et de $8 \%$ pour $\Delta h=2 \mathrm{~cm}$. Mais ceci n'explique pas le même «vieillissement»constaté sur le tronçon bitumineux, qui ne comporte pas de joint. Dans ce dernier cas, on peut émettre l'hypothèse d'ondulation de grande longueur d'onde directement sous l'influence du terrain.

Par ailleurs, nous avons également vérifié, bien que les essais aient été exécutés en l'absence totale de vent, que l'effort tranchant dû au vent à l'intersurface air-eau est négligeable.

\begin{tabular}{|c|c|c|c|c|c|}
\hline \multirow{2}{*}{ Grandeur } & \multirow{2}{*}{ SrMbole } & \multicolumn{2}{|c|}{1961} & \multicolumn{2}{|c|}{1962} \\
\hline & & Béton & Bitume & Béton & Bitume \\
\hline Débit. . . . . . . & $\mathrm{Qm} \mathrm{m}^{3} / \mathrm{s}$ & 166,8 & 166,8 & 163,9 & 163,9 \\
\hline Vitesse. . . . . . . . . . . . & $\mathrm{V} \mathrm{m} / \mathrm{s}$ & 1,66 & 1,65 & 2 & 2 \\
\hline Rayon hydraulique.......... & $\mathrm{R} \mathrm{m}$ & 3,45 & 3,46 & 3,085 & 3,125 \\
\hline Diamètre équivalent............. & $\mathrm{D} \mathrm{m}$ & 13,86 & 13,84 & 12,33 & 12,5 \\
\hline Nombre de Reynolds.......... & $\mathfrak{R}$ & $2,3.10^{7}$ & $2,3.10^{7}$ & $2,466.10^{7}$ & $2,5 \cdot 10^{7}$ \\
\hline Coefficient de Chézy.......... & $\mathrm{C}_{\mathrm{MTS}}$ & 84,7 & 77,2 & 80,03 & 69,79 \\
\hline Coefficient de Strickler............... & $\mathrm{K}_{\mathrm{MTS}}^{\mathrm{MTS}}$ & 69,6 & 63,9 & 66,31 & 57,71 \\
\hline Coefficient universel.................. & $\lambda \%$ & 1,09 & 1,316 & 1,22 & 1,605 \\
\hline Rugosité équivalente, suivant Powell. . . ... & $\varepsilon_{p} \mathrm{~mm}$ & 0,77 & 1,7 & 1,1 & 3 \\
\hline Rugosité équivalente, suivant Strickler. . . . . & $\varepsilon_{\mathrm{S}} \mathrm{mm}$ & 2,7 & 4,7 & 3,64 & 8,5 \\
\hline Rugosité équivalente, suivant Colebrook. . . . & $\varepsilon_{\mathrm{C}} \mathrm{mm}$ & 0,76 & 2,17 & 1,5 & 5,2 \\
\hline
\end{tabular}

Tableau IV 


\section{VIII. - CONCLUSION}

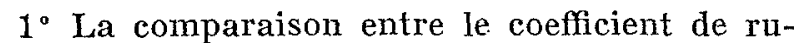
gosité correspondant aux deux tronçons bitume et béton, qui avait fait l'objet initial des essais, met en évidence un écart d'environ $10 \%$ en faveur du béton. Il est bon de rappeler à ce sujet la qualité tout à fait particulière du revêtement béton, du fait du procédé de bétonnage utilisé.

$2^{\circ}$ Les essais entrepris à quinze mois d'intervalle font apparaître une diminution de $10 \%$ environ sur le coefficient de rugosité pour chaque type de revêtement.

Nous n'avons pas pu déterminer les causes exactes de cet écart. $3^{\circ}$ Il serait, à notre point de vue, souhaitable que des essais semblables effectués avec le même souci de précision soient réalisés sur d'autres installations, ce qui ne présente guère de diffcultés à condition d'aménager le dispositif d'essais au cours des travaux de construction du canal.

En ce qui nous concerne, nous comptons, d'une part poursuivre les essais sur le canal de Palaminy et, d'autre part, entreprendre les mêmes essais sur le canal de l'aménagement de Saint-Julien, dont la mise en service est prévue pour 1966.

\section{DIS CUSSION}

président : M. Durfaut

M. le Président remercie $M$. Castex et ouvre la discussion en la divisant en deux parties.

- mesures absolues de pertes de charge sur un revêtement en béton;

- mesures relatives entre le revêtement en béton et ie revêtement bitumineux.

M. Willa tient à rendre hommage à la recherche de précision et à la qualité des résultats présentés par M. CASTEX. Ceux-ci sont d'autant plus intéressants que les mesures de pertes de charge en canal sont généralement difficiles, et de toute façon assez rares.

Il demande quelques éclaircissements sur les points suivants: tout d'abord quelques hypothèses paraissent avoir été faites au départ, elles sont relatives :

- à l'identité du rendement des deux turbines et à la conservation de celui-ci dans le temps (problèmes des conjugaisons, en particulier);

- à la conservation parfaite du tarage des contrôles Winter-Kennedy pendant 15 mois.

Par ailleurs :

- Le défaut de raccordement des deux troncons du canal peut-il être à l'origine d'une perte de charge singulière sensible?

- Comment expliquer que les points représentatifs de la ligne piézométrique sont parfaitement alignés dans le tronçon aval et mal dans le tronçon amont?

- Ce défaut d'alignement des points relatifs au tronçon amont est supérieur aux écarts aléatoires de mesure. Dans ces conditions, pourquoi négliger cer tains points dans le tracé de la droite représentative de la ligne piézométrique?

- Les points laissés à l'écart ne peuvent-ils traduire un remous liẻ au changement de section?

Quoi qu'il en soit, il est évident que, même en admettant une malchance telle que toutes les erreurs de mesure se soient composées défavorablement, l'évolution constatée dans le Strickler reste significative et il est surprenant a priori que cette évolution soit à peu près la même (10 et $12 \%$ ) dans les deux tronçons pourtant bien différents de nature et de réalisation.
M. Castex, en réponse à la question de M. WhLLM sur le tarage du contrôle piézométrique, indique que dans le cas particulier de Palaminy, l'exposant de la loi $Q^{n}$ est compris entre 1,98 et 1,99 ; c'est la raison pour laquelle pour le groupe 1 , on a pris le coefficient 2 .

Au cours de la deuxième série d'essais, on a repris la courbe complète de l'enveloppe des rendements des deux groupes et on a constaté que la différence de ces deux enveloppes n'excédait pas 0,4 ou $0,5 \%$. Les deux groupesi ont donc, à 0,4 ou $0,5 \%$ près, le même rendement.

Si on admet qu'il y ait une baisse de rendement entre la première et la deuxième série de mesures, il faudrait admettre que le rendement ait baissé simultanément de la même façon sur les deux groupes, ce qui ne semble pas possible, parce que les durées d'exploitation de chacun d'eux étaient nettement différentes durant les quinze mois. Les mesures de débits sont absolument indépendantes de la came de conjugaison.

En ce qui concerne le raccordement des deux tronçons, le calcul d'une perte de charge montre que la perte de charge singulière est négligeable.

M. Castex indique que la divergence des deux derniers points de mesure sur le tronçon bitumineux peut etre due à l'influence de l'orientation de la boite de prise de pression par rapport à la paroi. Les vitesses dans le canal sont de l'ordre de $2 \mathrm{~m} / \mathrm{s}$, ce qui correspond à $\left(\mathrm{V}^{2} / 2 \mathrm{~g}\right)=20 \mathrm{~cm}$. Si l'arasement de la boîte de prise de pression n'est pas parfait, il se peut que l'on récupère $10 \%$ du V $2 / 2 g$, soit $2 \mathrm{~cm}$.

Par ailleurs, en ce qui concerne le tracé de la ligne piézométrique, l'opération effectuée par une dizaine d'expérimentateurs a conduit à la même droite moyenne, compte tenu du fait que les lignes représentatives ne peuvent présenter rle discontinuité au raccordement des deux tronçons.

Malheureusement, il est évident que la longueur de $400 \mathrm{~m}$ pour le tronçon bitumineux est un peu faible. On n'a pas pu obtenir d'avoir un tronçon de $600 \mathrm{~m}$ comme il avait été demandé. 
M. le Président repose la question posée par M. CasTex dans son rapport: A quoi doit-on attribuer la variation du coefficient ou de la perte de charge en fonclion du temps?

M. WiLLM rappelle à ce sujet un exemple de perte de charge un peu du même ordre :

Un rapprochement peut etre fait entre l'augmentation des pertes de charge du canal de Palaming el un phénomène du même ordre, quoique plus important, apparu dans la galerie de Monceau-la-Virole peu après la mise en service de cet aménagement; cetle galerie, dont les parois revêtues présentaient un excellent état de surface, a vu ses pertes de charge passer de $6 \mathrm{~m}$ à $12 \mathrm{~m}$ environ en quelques mois. Le phénomène, dabord mystérieux, a pu être attribué à la prolifération d'une algue vivant en symbiose avec des bactéries du fer, et aynt pour effet d'augmenter la rugosité des surfaces (la réduction de section restant négligeablel. La perte d'énergie correspondante était de lordre de 1 million de $\mathrm{kTh}$ par an: elle justifiait des grattages périodiques et lat mise en route d'études ayant pour objectif la destruction de ces organismes.

Une petite station experimentale avait donc été montée à proximité de l'usine de Moneean eq allait commencer à fonctionner quand la prolifération des algues s'arrêta spontanément.

Cependant, il a été possible de se rendre compte que ces micro-organismes se developpaient electivement dans les eaux granitiques du Massif Central, à faible pH et tout particulièrement dans les installations récemment mises en service, les produits de décomposition des éléments organiques noyés dans les retenues modifiant sans doute favorablement le milieu.

M. WILLM projette deux microphotographies montrant des colonies filamenteuses de «sideris capsa » en association avec des algues monocellulaires provenant de prélèrements faits à l'époque à Monceau-la-Virole.

$M$ Ie président remercie $M$. Willm et espère que celui-ci a donné une orientation aux recherches futures, puisque lon compte bien essayer de tirer au clair cette question de perte de charge.

M. Duranton suggère à M. Castex de s'assurer, à tout hasard, que les parois du canal dans les tronçons intéressés n'ont subi aucune déformation entre les deux séries de mesures.

De telles deformations pourraient se traduire notamment par de faibles ondulations du revêtement bitumineux ou par de légers décalages aux joints du revêtement béton et affecteraient le réglage d'ensemble de la paroi.

M. Castex indique que le prochain assèchement du canal permettra de vérifier ce point.

M. Mathaly ajoute que l'on n'a observé aucune onduiation des bajoyers, ce qui se verrait facilement en observant la surface à l'œil nu.

M. Ie Président remercie M. CASTex et souhaite qu'avec la collaboration d'E.D.F. et en particulier la D.T.G., il arrive à déterminer les causes de ce qu'il a indiqué.

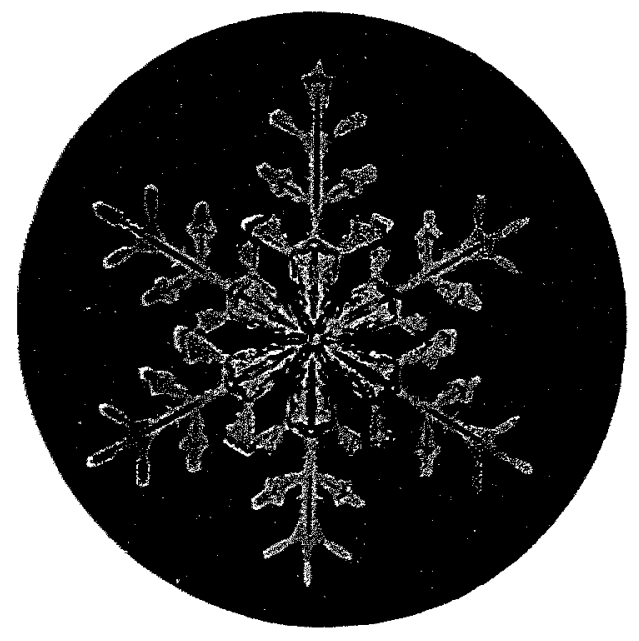

